\title{
An Audiovisual Wireless Field Guide
}

\author{
Ruben Gonzalez ${ }^{1,2}$ and Yongsheng Gao ${ }^{1,2}$ \\ ${ }^{1}$ Queensland Research Laboratory, National ICT Australia, QLD 4072, Australia \\ ${ }^{2}$ Institute for Integrated and Intelligent Systems, Griffith University, Australia
}

\begin{abstract}
This paper describes our work on developing a multimedia wireless field guide platform (WFG) for both fully automatic and assisted identification of different fauna and flora species. Built using a smart-client and server model it supports both visual and acoustic capture of individual specimen samples with optional graphical annotation for posing queries alongside more traditional searching and browsing query interfaces. The WFG assists the user to iteratively converge on a correct match by seeking additional information required to resolve class uncertainty.
\end{abstract}

\section{Introduction}

Over the last decade technological advances have radically increased the availability of data processing. Once confined to offices and laboratories, there is now sufficient computing power in a typical mobile telephone handset to rival that of computer workstations from some years ago. This ability to undertake data processing in the field is greatly enhanced by wireless communication capabilities that provide remote access to a virtually unlimited amount of remote computing resources and data. Thus computing can now be applied in new ways to challenging problems that previously would have been impractical.

One such area is species identification in the field that, in the case of some species, can be a difficult task for biologists let alone the general public. Field guides that can automatically identify or interpret data for different species would provide a significant benefit to field workers in areas of conservation of threatened species, biosecurity with regard to pests and invasive species, and other key areas.

Current paper field guides are being gradually replaced with their digital counterparts that can be accessed using electronic devices such as handheld computers [1]. These require users to locate information about different species by searching through scientific or common names, or by browsing through pictures using tools similar to Photomesa [2]. There are also extensive efforts to digitise and catalogue taxonomic and biodiversity information regarding different species and other efforts within the scientific communities to ensure interoperability between these databases by various organisations such as the Global Biodiversity Information Facility [3], the Biodiversity Information Standards (TDWG) group [4] and the National Biological Information Infrastructure (NBII) [5]. Given that there are nearly two million identified species representing a total of less than ten percent of the total 
number of species on earth [6] the large amount of data only compounds the problem of identifying an unknown species.

One solution used by computer-based guides such as Lucid [7], DiscoverLife [8] and Delta [9] permits filters to be applied to various observable or deductible properties to narrow down the search such as selecting all birds with red wings and long beaks. This approach doesn't provide automated species classification or recognition but, rather, guides the user to possible species. It relies on ordering the distinguishing characteristics of each species in databases so as to most rapidly converge on a particular species using principles derived empirically from experts (and not by the use of technologies such as Decision Trees [10] to automatically order the discriminating features).

Alternatively one could make use of Pattern Recognition and Machine Learning algorithms to automatically identify species and interpret information collected in the field, in situ. These can either require little or no domain knowledge such as those used in generic content-based retrieval [11], or, be highly domain specific such those used to identify specific leaf shapes of terrestrial [12] or aquatic plants [13]. If successful, these approaches return a ranked short-list of potential matches for users to choose from as the most likely identification.

The content-based retrieval methods return matching images based on statistical similarity of pixel data and don't work very well in distinguishing images with similar statistical distributions but different local or structural features such as differences in the shape of a wing shape for example. Alternatively, domain specific methods ignore the bulk statistical properties and look for the fine discriminating details in the data, which if not found, or if they don't conform within an expected range prohibits the matching process from continuing.

One of the problems with the application of Pattern Recognition techniques to identification is that success is highly dependent on whether key discriminating features have been captured with sufficient quality. If they have not low quality matches will be presented to the user. Hence instead of just short-listing potential matches and leaving it up to the user to decide which was the correct one, we wanted to use automatic pattern recognition to interactively assist users in choosing the correct match if the WFG system was not able to confidently identify a species on its own. The remainder of this paper describes the design of a WFG and its approach to automatic species identification including its query interface and system architecture.

\section{Query Interface}

The WFG supports three primary query interfaces; searching by browsing, searching through text and key matching and query by example. For text and key based searching the WFG backend essentially operates as a proxy for existing taxonomic databases. Browsing type queries are performed through a visual taxonomic hierarchy. Users are initially presented with a pictorial representation of the top-level of this hierarchy, consisting of selected phyla and they can navigate down through the various taxonomic ranks by clicking on images at each level. 
In the case of query by example the query data is first captured using an appropriate capture device connected to the WFG client device such as digital microscopes, cameras and microphones. Once the relevant data has been captured it can be manually annotated or cropped to identify specific regions of interest using the built-in tools as is depicted in Figure 1. It can then be submitted for identification by one of an arbitrary number of server side automatic identification modules (AIMs). The captured data and annotations are forwarded to the processing server that passes it on to the appropriate AIM. If identification can be successfully performed, the module returns a resulting identifier, which is typically the entity's scientific name, to the server that then uses it as a text search key for a relevant taxonomic database.

Supporting multiple modules permits specialised AIMs to be developed for identifying individual species within a given genus, family, order, class or phylum. When the broad level taxonomic classification is unknown a generic AIM is first used to resolve the taxonomic classification sufficiently for the system to select the appropriate specialised module to perform the species identification.

In the case where a module is unable to successfully identify a species an AIM can request additional information from the user by creating an interactive or "assisted" query session to help guide it towards identity resolution. AIMs can request one of three different types of assistance; annotation, selection and identification. In situations where the AIM is unable to distinguish the salient attributes required to identify a specific species, it can request the user to annotate the location of the required feature. In cases where a module can distinguish important features but due to occlusion or camera angle, cannot recognise other characteristics it needs to uniquely resolve identity it can request the end user to select the appropriate characteristic from a set of candidates. As a trivial example it may present to the user a selection of wing shapes.

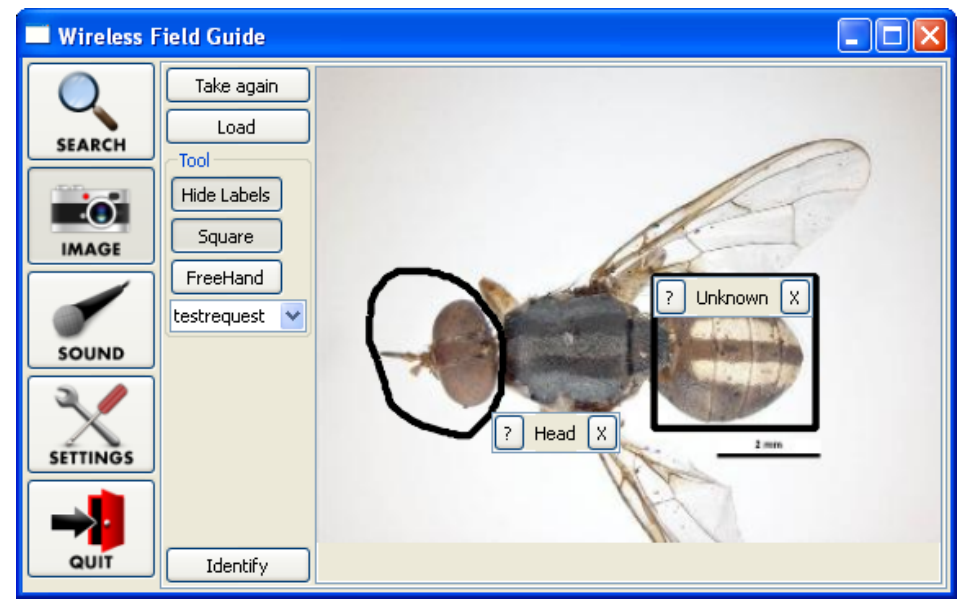

Figure 1 Image Capture and Annotation Screen (Fruit fly)

Once an AIM has successfully isolated the characteristic features of a specimen it can either identify the species directly itself or it can pass the features to a character 
database for identification. These options and the interaction process of a query are shown in Figure 3.

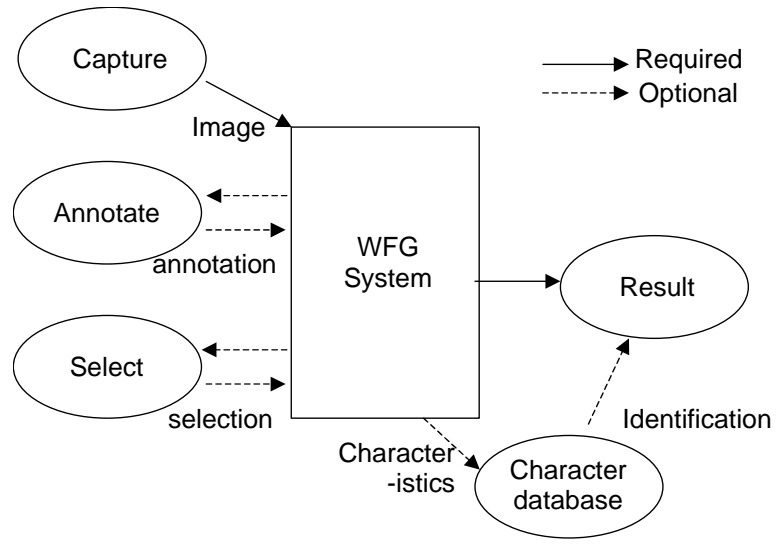

Figure 1 Image Query Interaction Process

\section{System Architecture}

Unlike other efforts that focus on taxonomic or character database architectures [14], our approach primarily focuses on providing a flexible platform for capturing specimen data and automatically extracting and recognising their features either directly or by leveraging existing taxonomic and biodiversity databases. We also wanted the WFG system to be transparently upgradeable to identify additional species from additional phyla or to make use different identification techniques without requiring any alterations to software on the field devices. Other requirements were to make the WFG system ubiquitously accessible using as wide a range of wireless client devices as possible. This included devices with an inbuilt ability to natively capture images and sound and as those without and to also support the use of other capture devices such as microscopes.

Currently supported field devices for the WFG include an N97 that uses the Nokia Symbian 6.0 platform and a Viliv S5 handheld computer running Microsoft Windows. WFG smart client software running on these devices provides audio, image and location (GPS coordinates) capture and graphical annotation functions and xHTML forms support for framing queries that are submitted to the server over HTTP 1.1. It also displays responses from the server using a version of XHTML basic 1.1.

The WFG server is predominantly a two-tier architecture. The first tier contains the presentation and high-level application logic. The second tier consists of AIMs and various databases. The web server in the first tier is responsible for communicating with the client and deciding whether to pass query data to selected taxonomic databases in the case of query by searching or by visual browsing or alternatively to an appropriate AIM.

In the simplest case where query data is passed to an AIM the response provided by the AIM to the web server is either the scientific name of a species or the name of 
a higher order taxonomic classification. This is respectively subsequently submitted to a taxonomic database to retrieve textual, visual or acoustic information of the identified species to return to the field device client or to another appropriate AIM for further identification, as per Figure 2.

Each AIM performs a number of tasks. It first attempts to extract from the query by example data the characteristic features for distinguishing between various species. If it is successful these are individually classified into one of various potential classes using machine-learning techniques. Each class corresponds to different characteristics that differentiate various species. The name of the species that matches the specific combination of these characteristics is the result returned to the server. In performing these tasks the AIM must communicate with a database containing feature vectors for each characteristic of known species. This database can be embedded within the AIM, or it can be external to the module as a separate database.

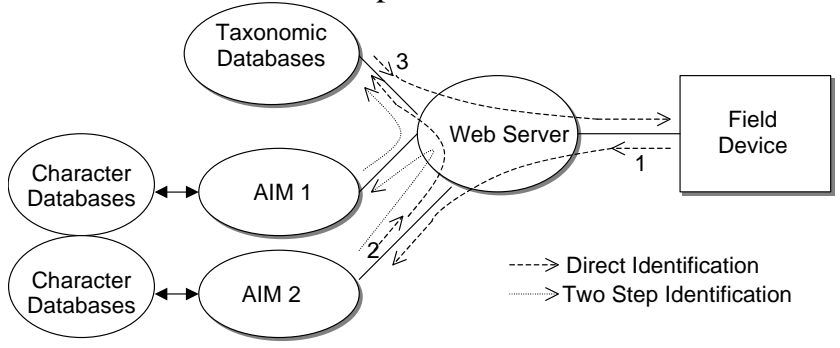

Figure 2 Information Flow

The AIMs themselves are executable objects that are invoked by the server. Once AIMs are invoked by a new query they persist through a user session until the query is resolved or until they time out. AIMs communicate with the server using a library of software routines (WFGLib) that manage the data exchange and encode and decode any image, audio or annotation data appropriately. AIMs can be added or deleted transparently from the WFG system via a simple web interface. Thus AIM developers can focus on implementing identification algorithms rather than communication and system integration issues. The next sections discuss some the methods used by generic AIMs for species identification.

\section{$4 \quad$ Audio Identification}

Automatic identification of animals by their calls has been an area of study over the last decade. This includes identification of bird and frog calls $[15,16,17,18]$, bat echolocation calls [19, 20] and insects such as grasshoppers and cicadas [21]. These studies have made use of a variety of machine learning techniques such as C4.5 decision trees [22], as well as Neural Networks [23] and classification methods including k-Nearest Neighbour, Gaussian Mixture Models and Support Vector Machines (SVM) [24, 15].

Audio classification approaches typically use a combination of temporal features and spectral features [25] derived from the short term Fourier transform or Mel- 
Frequency Cepstral Coefficients (MFCCs) [26]. Some of the more common temporal features include the signal bandwidth (BW), spectral centroid (SC), short-term energy (E), energy flux (F), Zero Crossing Rate (ZCR), and pitch (P) by means of subharmonic summation [27]. The most common spectral features are the first six MFCCs, which are calculated as the cepstrum of the mel-warped spectrum.

Generic audio AIMs in the WFG currently utilise this set of features and a k-NN classifier which while being relatively simple can perform within a few percent of the more sophisticated classifiers. For evaluation we have used a database of 1629 recordings of seventy four different species of native Australian frogs from the Myobatrachidae family (Southern Frogs). Using tenfold cross validation we achieved over $90 \%$ correct classification with single and multiple calls in the laboratory. We also used a database of sounds from 381 different species of insects with which we were able to also achieve a correct classification into insect families of over $90 \%$.

In practice the identification of single isolated animal calls under ideal conditions is fairly easy and close to $90 \%$ correct performance can be achieved. In the field, with high levels of background noise, compounded by simultaneous calls from different species [22] identification is less successful. In one study, a success rate of only about $60 \%$ was achieved [28] in the in-field identification of twenty different species of frogs. This is further compounded in the case of the WFG since in the interests of reducing network latency and other costs the captured audio data will typically be compressed before being communicated to the server for identification. While the effects of background noise on the classification performance are variable the effects of audio compression is more predictable. To determine what effect audio compression has on identification accuracy we performed some experiments using both the ADPCM [29] and MP3 algorithms as being representative of a range of audio compression methods as is reported in [30]. We discovered as is shown in Figure 3 that using ADPCM encoding with 4 bits per sample resulting in a consistent drop of about $3 \%$ in classification accuracy irrespective of analysis window size. This drop is due to the introduction of quantization noise.

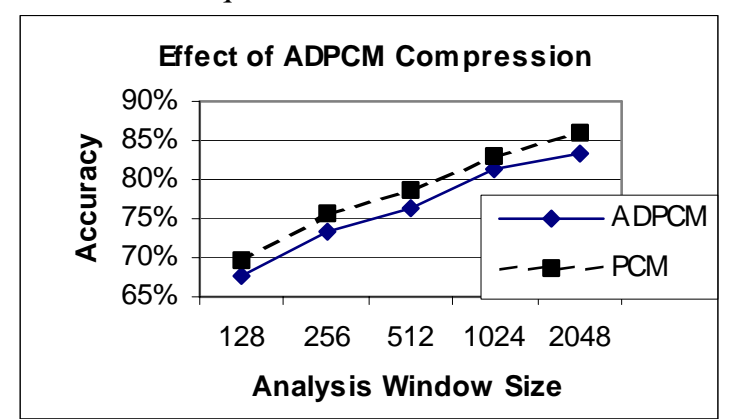

Figure 3. Compressed Audio Classification Performance

In the case of the MP3 compression identification performance was notably worse and varied depending on the analysis window size used for extracting classification features as is shown in Figure 4. 


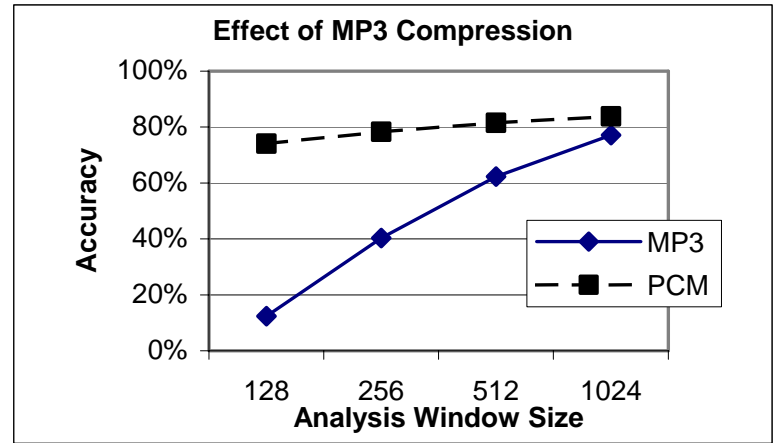

Figure 4. MP3 Compressed Classification Performance

\section{Image Identification}

Existing work on image-based identification for electronic field guides has largely focused on plants. These have made use of Fourier Shape Descriptions [31] other means to characterise the two dimensional shapes of leaves in rotation and scale invariant ways. In entomology as with other fields within zoology the shape and contour information of a species is often of primary importance in differentiating it from others. The shape is obtained from edge information that can be directly computed from spatio-chromatic gradient operators or indirectly via combining gradient information from each colour source.

Our approach for image identification is to analyse the colour or texture related features in annotated regions together with the local shape of the object. We represent the shape using line edge maps [32] that are created from edge contours using the Canny Edge Detector. This permits shape similarity to be measured using a modified Hausdorff Distance. This shape information is combined with micro-pattern representations of image texture defined by the Local Derivative Pattern (LDP) [33]. The LDP encodes the higher-order derivative texture pattern which contains more detailed information than that the first-order Local Binary Pattern (LBP) [34] can extract. The $n^{\text {th }}$-order LDP is a sequence of binary digits encoding derivative changes in a local region of $(n-1)^{\text {th }}$-order derivative images $I_{\alpha}^{n-1}(Z)$ as

$$
L D P_{\alpha}^{n}\left(Z_{0}\right)=\left\{f\left(I_{\alpha}^{n-1}\left(Z_{0}\right), I_{\alpha}^{n-1}\left(Z_{1}\right)\right), f\left(I_{\alpha}^{n-1}\left(Z_{0}\right), I_{\alpha}^{n-1}\left(Z_{2}\right)\right), \cdots, f\left(I_{\alpha}^{n-1}\left(Z_{0}\right), I_{\alpha}^{n-1}\left(Z_{8}\right)\right)\right\},
$$

where $I_{\alpha}^{n-1}\left(Z_{0}\right)$ denotes the $(n-1)^{\text {th }}$-order derivative in $\alpha$ direction at $Z=Z_{0}$. $f\left(I_{\alpha}^{n-1}\left(Z_{0}\right), I_{\alpha}^{n-1}\left(Z_{i}\right)\right)$ encodes the $(n-1)^{\text {th }}$ order gradient changes into a binary digit as follows:

$$
f\left(I_{\alpha}^{n-1}\left(Z_{0}\right), I_{\alpha}^{n-1}\left(Z_{i}\right)\right)=\left\{\begin{array}{ll}
0, & \text { if } I_{\alpha}^{n-1}\left(Z_{i}\right) \cdot I_{\alpha}^{n-1}\left(Z_{0}\right)>0 \\
1, & \text { if } I_{\alpha}^{n-1}\left(Z_{i}\right) \cdot I_{\alpha}^{n-1}\left(Z_{0}\right) \leq 0
\end{array}, \quad i=1,2, \cdots 8 .\right.
$$

This binarization creates an additional order of texture information within the neighbourhood. The texture-pattern representation of each region of interest in the 
captured images is then classified against known micro-patterns that are particular to specific species.

\section{Conclusions and Further Work}

This paper has described our work on developing a multimedia wireless field guide that provides automatic acoustic as well as visual identification. Unlike conventional image based approaches that attempt to identify an image of a specimen as a single class in a multidimensional feature space following the classical image content-based retrieval model our approach leverages existing character databases model where the identification is based on classifying individual visual distinguishing characteristics of specimens. As this approach requires such characteristics to be visible in query images the query interface supports the notion of an assisted query session whereby the user can be prompted to provide additional information that may be not be apparent in the query image but is necessary for correct identification. Our initial focus is visual identification of insects and acoustic identification of frogs.

Since our approach leverages existing character databases our focus has been on the processing model and software architecture to facilitate ubiquitous mobile access. The processing model revolves around the need for specialized automatic identification modules (AIM) for different taxonomic groups since different characteristics are required for identifying species belonging to different groups. Since the appropriate AIM for an identification query may be unknown a hierarchical process of incremental refinement is pursued utilizing high-level classification AIMs to converge on the final identification.

While the WFG system implementation is complete further work is required on developing the individual AIMs. For the acoustic AIMs this includes investigating ways to improve performance in the field by reducing noise sensitivity and resolving simultaneous call confusion. For the visual identification AIMs further work involves modeling and implementing AIMs for locating the visual characteristics in different query images classes from which to extract LDPs and LEMs for classification.

\section{$7 \quad$ Acknowledgements}

The authors would like to acknowledge the assistance NICTA for providing funding for this work and the efforts of Steven Tucker, Craig Hume and Coby Chapple in the development of the WFG client-server software.

\section{References}

[1] Stevenson, R. D., W. A. Haber, and R. A. Morris. 2003. "Electronic field guides and user communities in the eco-informatics revolution.” Conservation Ecology 7(1): 3. [online] URL: http://www.consecol.org/vol7/iss1/art3/ 
[2] White, S., Marino, D., and Feiner, S., "Designing a Mobile User Interface for Automated Species” Identification, Proc. ACM CHI 2007, April 28-May 3, 2007, pp. 291-294.

[3] Global Biodiversity Information Facility, http://www.gbif.org [accessed 2010].

[4] Biodiversity Information Standards, http://www.tdwg.org/ [accessed 2010]

[5] National Biological Information Infrastructure, http://www.nbii.gov [accessed 2010].

[6] May, R. M. (1990). How many species? Philosophical Trans. of the Royal Society of London. B, 330, 293-304.

[7] http://www.lucidcentral.com/ [accessed 2010].

[8] http://www.discoverlife.org/ [accessed 2010].

[9] Dallwitz, M. J. 1980. A general system for coding taxonomic descriptions. Taxon 29: 41-6.

[10] J. R. Quinlan, Learning decision tree classifiers, ACM Computing Surveys (CSUR), v.28 n.1, p.71-72, March 1996

[11] Datta, Ritendra; Dhiraj Joshi, Jia Li, James Z. Wang (2008). "Image Retrieval: Ideas, Influences, and Trends of the New Age". ACM Computing Surveys 40: 1-60.

[12] Agarwal, G., Belhumeur, P., Feiner, S., Jacobs, D., Kress, W.J., Ramamoorthi, R., Bourg, N., Dixit, N., Ling, H., Mahajan, D.,Russell, R., Shirdhonkar, S., Sunkavalli, K., and White, S. "First Steps Toward an Electronic Field Guide for Plants. Taxon, Journal of the International Assoc. for Plant Taxonomy”, 55(3), August 2006, pp. 597610

[13] Park J-K., Hwang E., "Implementation of an Aquatic Plant Information Bank" Proceedings of the 2007 Frontiers in the Convergence of Bioscience and Information Technologies, pp. 861-866, ISBN:978-0-7695-2999-8

[14] R.A. Morris, R.D. Stevenson and W.Haber "An architecture for electronic field guides”, Journal of Intelligent Information Systems, V.29, No.1, August, 2007 pp.97110

[15] M.A. Acevedoa, C.J. Corrada-Bravoc, H. Corrada-Bravob, L. J. Villanueva-Riverad and T. Mitchell Aidea, "Automated classification of bird and amphibian calls using machine learning: A comparison of methods” Ecological Informatics, Vol. 4, Issue 4, Sept. 2009, Pages 206-214.

[16] Mills H.1995. Automatic detection and classification of nocturnal migrant bird calls. J Acoust Soc Amer. 97: 3370-3371.

[17] H.Wang, J.Elson, L.Girod, D.Estin and K.Yao, "Target Classification and Localization in Habitat Monitoring", In Proc. of IEEE International Conference on Acoustics, Speech, and Signal Processing (ICASSP 2003) Hong Kong.

[18] Darren Moore. "Demonstration of bird species detection using an acoustic wireless sensor network." 33rd IEEE International Conference on Local Computer Networks (LCN 2008) : SenseApp 2008; Montreal, Que. IEEE; 2008: 730-731. ISBN: 9781424424122

[19] Parsons S.2001. Identification of New Zealand bats in flight from analysis of echolocation calls by artificial neural networks. J Zool London 253: 447-456.

[20] Vaughan N, Jones $G$ and Harris S.1997. Identification of British bat species by multivariate analysis of echolocation call parameters. Bioacoustics 7: 189-207. 
[21] Ohya Eand Chesmore ED.2003. Automated identifica-tion of grasshoppers by their songs. Iwate University, Morioka, Japan: Annual Meeting of the Japanese Society of Applied Entomology and Zoology.

[22] A.Taylor, G.Watson, G.Grigg and H.McCallum, "Monitoring Frog Communities: An application of Machine Learning", in the Proceedings of 8th innovative applications of AI conference (IAAI) Portland, Oregon 1996, pp.1564-1569

[23] Yen, Gary G.; Fu, Qiang, "Automatic frog call monitoring system: a machine learning approach" Proc. SPIE Vol. 4739, p. 188-199, Applications and Science of Computational Intelligence V, Kevin L. Priddy; Paul E. Keller; Peter J. Angeline; Eds, 2002

[24] Chenn-Jung Huang, Yi-Ju Yang, Dian-Xiu Yang, "Frog classification using machine learning techniques", Expert Systems with Applications: An International Journal Volume 36 , Issue 2 (March 2009) Pages 3737-3743 ISSN:0957-4174

[25] R. Gonzalez, "Better than MFCC Audio Classification Features” Submitted to The 2011 Pacific-Rim Conference on Multimedia (PCM 2011) December 20-22, Sydney, Australia

[26] Peltonen, V. Tuomi, J. Klapuri, A. Huopaniemi, J. Sorsa, T., “Computational auditory scene recognition”, Proceeding of. International Conference on Acoustics, Speech, and Signal Processing, (ICASSP 2002). IEEE, May 13-17, 2002, Orlando, FL, USA, vol.2, pp:1941-1944

[27] D.J.Hermes, “Measurement of pitch by subharmonic summation” J. Acoust. Soc. Am. Volume 83, Issue 1, pp. 257-264 (January 1988)

[28] Ning-Han Liu , Chen-An Wu and Shu-Ju Hsieh, Long-Term Animal Observation by Wireless Sensor Networks with Sound Recognition, WASA 2009, LNCS 5682, p.1-11, 5th Aug 2009

[29] ITU Recommendation G.726, “40, 32, 24, 16 kbit/s Adaptive Differential Pulse Code Modulation (ADPCM)"

[30] R.Gonzalez, "Effects of Compression and Window Size on Remote Acoustic Identification using Sensor Networks” Proceedings of ICSPCS'2010. pp.

[31] Belhumeur, P.N., Chen, D., Feiner, S., Jacobs, D.W., Kress, W.J., Ling, H.B., Lopez, I., Ramamoorthi, R., Sheorey, S., White, S., Zhang, L., "Searching the World's Herbaria: A System for Visual Identification of Plant Species," Proceedings of the 10th European Conference on Computer Vision, Marseille, France, October 12-18, 2008 (ECCV 2008, IV: 116-129)..

[32] Y. Gao and M. Leung, “Face Recognition Using Line Edge Map”, $\underline{\text { IEEE Transactions }}$ on Pattern Analysis and Machine Intelligence, Vol. 24, No.6, pp.764-779, 2002.

[33] B. Zhang, Y. Gao, S. Zhao and J. Liu, "Local Derivative Pattern versus Local Binary Pattern: Face Recognition with High-Order Local Pattern Descriptor”, IEEE Transactions on Image Processing, Vol. 19, No. 2, pp. 533-544, 2010.

[34] T. Ojala, M. Pietikäinen, and T. Mäenpää, "Multiresolution Gray-Scale and Rotation Invariant Texture Classification with Local Binary Patterns," TPAMI, 24(7): 971-987, 2002. 